\title{
Water corrosion of spent nuclear fuel: radiolysis driven dissolution at the $\mathrm{UO}_{2} /$ water interface
}

\author{
Ross Springell, ${ }^{\text {*a }}$ Sophie Rennie, ${ }^{a}$ Leila Costelle, ${ }^{a}$ James Darnbrough, ${ }^{a}$ \\ Camilla Stitt, ${ }^{a}$ Elizabeth Cocklin, ${ }^{\mathrm{b}}$ Chris Lucas, ${ }^{\mathrm{b}}$ Robert Burrows, ${ }^{\mathrm{c}}$ \\ Howard Sims, ${ }^{d}$ Didier Wermeille, $\dagger^{\mathrm{e}}$ Jonathan Rawle, ${ }^{f}$ Chris Nicklin, ${ }^{f}$ \\ William Nuttall, ${ }^{g}$ Thomas Scott ${ }^{a}$ and Gerard Lander ${ }^{\mathrm{h}}$
}

Received 9th December 2014, Accepted 28th January 2015

DOI: $10.1039 / \mathrm{c} 4 \mathrm{fd} 00254 \mathrm{~g}$

$X$-ray diffraction has been used to probe the radiolytic corrosion of uranium dioxide. Single crystal thin films of $\mathrm{UO}_{2}$ were exposed to an intense $\mathrm{X}$-ray beam at a synchrotron source in the presence of water, in order to simultaneously provide radiation fields required to split the water into highly oxidising radiolytic products, and to probe the crystal structure and composition of the $\mathrm{UO}_{2}$ layer, and the morphology of the $\mathrm{UO}_{2}$ /water interface. By modeling the electron density, surface roughness and layer thickness, we have been able to reproduce the observed reflectivity and diffraction profiles and detect changes in oxide composition and rate of dissolution at the Ångström level, over a timescale of several minutes. A finite element calculation of the highly oxidising hydrogen peroxide product suggests that a more complex surface interaction than simple reaction with $\mathrm{H}_{2} \mathrm{O}_{2}$ is responsible for an enhancement in the corrosion rate directly at the interface of water and $\mathrm{UO}_{2}$, and this may impact on models of long-term storage of spent nuclear fuel.

\footnotetext{
anterface Analysis Centre, University of Bristol, Bristol BS2 8BS, UK. E-mail: phrss@bristol.ac.uk; Tel: +44 (0) 1173311176

${ }^{b}$ Department of Physics, University of Liverpool, Liverpool L69 7ZE, UK

${ }^{c}$ National Nuclear Laboratory, 102B Stonehouse Park, Sperry Way, Stonehouse, Gloucester GL10 3UT, UK ${ }^{d}$ National Nuclear Laboratory, Harwell Science and Innovation Campus, Oxfordshire OX11 OQT, UK ${ }^{\circ} X M a S$, European Synchrotron Radiation Facility, BP220, F-38043 Grenoble Cedex 09, France ${ }^{f}$ Diamond Light Source, Harwell Science and Innovation Campus, Harwell OX11 ODE, UK ${ }^{g}$ Department of Engineering and Innovation, The Open University, Venables Building, Milton Keynes MK7 6AA, $U K$

${ }^{h}$ European Commission, Joint Research Centre, Institute for Transuranium Elements, Postfach 2340, D-76125 Karlsruhe, Germany

$\dagger$ Alternative address: Department of Physics, University of Liverpool, Liverpool L69 7ZE, UK.
} 


\section{Introduction}

The future storage of spent nuclear fuel (SNF) poses some of the most challenging scientific and economic questions. ${ }^{1-3}$ With the growing consensus that storage of this material in a deep underground repository is the most viable long term solution, and the likely scenario of containment failure and groundwater contact, ${ }^{4,5}$ one of the central problems is to understand the reactions at the interface of the fuel with its surroundings. ${ }^{4,6-8}$ Of these, of course, water is the most important, as it can transport radioactive material away from the fuel repository and into the ecosystem. ${ }^{9}$

The predominant component of this fuel is a ceramic oxide of uranium, $\mathrm{UO}_{2}$, whose solubility in water of its stoichiometric U(Iv) form, is very low. ${ }^{\mathbf{1 0}}$ However, post burn-up, the $\mathrm{UO}_{2}$ fuel possesses levels of activity from $10^{14} \mathrm{~Bq}$ to $10^{16} \mathrm{~Bq}$ at its surface, depending on the reactor type, and this decays by approximately four orders of magnitude over the first 10000 years of proposed storage, at which point containment failure becomes significantly likely. ${ }^{11}$ The strong alpha, beta and gamma radiation fields are sufficient to radiolyse (radiolysis is the dissociation of molecules due to nuclear radiation) water in close proximity., ${ }^{\mathbf{4 2 , 1 3}}$ The radiolysis products comprise short-lived, highly oxidising free radical species, such as ${ }^{\circ} \mathrm{OH}$ and the much longer lived hydrogen peroxide molecule, $\mathrm{H}_{2} \mathrm{O}_{2} \cdot{ }^{14,15}$ Under these highly oxidising conditions it is possible to form $\mathrm{UO}_{2}{ }^{2+}$ (uranyl) ions via the oxidation of $\mathrm{U}(\mathrm{IV})$ to $\mathrm{U}(\mathrm{VI})$, whose solubility in water is several orders of magnitude greater than $\mathrm{UO}_{2},{ }^{6}$ leading to accelerated dissolution of the fuel matrix and potential release of radionuclides into the environment. Therefore, an understanding of the water $/ \mathrm{UO}_{2}$ interface, and the ability to predict its long-term behaviour are vitally important.

A stored spent fuel pellet possesses a great deal of complexity, including defects, He bubbles, microscopic cracking and fission daughter products for example. The situation becomes even more complicated when one includes groundwater, containing various ionic species, and further still, if one includes the potential influence from the cladding material. To date, the majority of studies of this complex system have focused on the chemical composition of the dissolution and the electrochemistry of the corrosion mechanism. ${ }^{\mathbf{4 , 7 , 9 , 1 0 , 1 6}}$ Since one of the most important factors driving oxidising conditions in the groundwater is the radiolysis of the water, ${ }^{6,17}$ some studies have gone further and have attempted to replicate the radiolytic conditions electrochemically, ${ }^{18,19}$ using external and dopant alpha sources ${ }^{9,20-22}$ and by the addition of $\mathrm{H}_{2} \mathrm{O}_{2}$ to the groundwater solution. ${ }^{6,10,23}$ This final method is hotly debated amongst research groups, since it is not clear precisely what the $\mathrm{H}_{2} \mathrm{O}_{2}$ concentration would be at the fuel surface.

Here, we report a new approach; we aim to remove much of the material complexity and study the corrosion of $\mathrm{UO}_{2}$ in pure water in the presence of strong radiation fields. Using high-quality single-crystal thin films of $\mathrm{UO}_{2}$ with atomically smooth surfaces and a thin layer surface tension cell of MilliQ, pure water (nominally pH 7), we expose this model fuel/groundwater interface to an intense, monochromated beam of X-rays from a synchrotron source; an approach first employed on the XMaS beamline, BM28 at the ESRF. This source-probe method allows us to simultaneously provide strong radiation fields and probe the 
structure of the interface. Using a combination of X-ray reflectivity (XRR) and high angle diffraction (XRD) in a specular geometry we are able to probe changes in the interface structure, roughness, electron density, crystallinity and eventual dissolution as a function of exposure time.

It is important to stress the surface sensitivity of these techniques (XRR and $\mathrm{XRD}$ ), and ask why this has not been observed previously with bulk $\mathrm{UO}_{2}$ samples. For typical energies and angles of incidence, the penetration of such a photon beam into $\mathrm{UO}_{2}$ is in the order several microns. Hence, there will be only a minuscule change in the Bragg reflected intensity, since $>99.5 \%$ of the intensity comes from the undisturbed $\mathrm{UO}_{2}$ bulk sample. Even if a single-crystal is used, no measurable change will be observed. On the other hand, by using an epitaxial film of $50 \AA$, there will be enough intensity (at a synchrotron) and the changes will be very substantial, often exceeding $50 \%$. There are also additional features in the diffraction profiles, resulting from finite thickness effects that give even more detailed information about the morphology of the interface. Such films therefore give unprecedented sensitivity to structural changes at the $\mathrm{UO}_{2}$ interface.

\section{Experimental}

Single-crystal thin films of $\mathrm{UO}_{2}$ were grown in a dedicated DC magnetron sputtering facility at the University of Bristol under UHV conditions. Samples were deposited in the three high symmetry directions, [001], [110] and [111], although the majority of the work presented here describes data collected from an [001]oriented $\mathrm{UO}_{2}$ sample, deposited onto a single-crystal [001]-YSZ (yttria-stabilised zirconia) substrate of dimensions $1 \mathrm{~cm} \times 1 \mathrm{~cm} \times 0.5 \mathrm{~mm} .{ }^{24}$ Reactive sputtering was used to deposit uranium in an argon pressure of $\rho_{\mathrm{Ar}}=7.2 \times 10^{-3} \mathrm{mbar}$ and an oxygen partial pressure of $\rho_{\mathrm{O}_{2}}=2 \times 10^{-5} \mathrm{mbar}$, to give a sputtering rate of $1.2 \AA_{\mathrm{UO}_{2}} \mathrm{~s}^{-1}$ in order to produce a sample of nominal thickness, $t_{\mathrm{UO}_{2}}=40 \AA$. Substrate heating was used to elevate the growth temperature to $\sim 550{ }^{\circ} \mathrm{C}$, providing thermal energy to improve the crystalline quality, monitored using in situ reflection high-energy electron-diffraction (RHEED).

Several samples were grown in order to verify the reproducibility of the experiment, and these were characterised using X-ray reflectivity (XRR) and high angle X-ray diffraction (XRD) on a Philips X'Pert Pro MRD, with a $\mathrm{Cu} \mathrm{K} \alpha$ source $(\lambda=1.54 \AA)$. This same geometry was used in the synchrotron measurements, carried out at the XMaS beamline, BM28, ESRF ${ }^{25}$ and I07, Diamond Light Source, as shown in Fig. 1.

$\mathrm{X}$-ray reflectivity is a non-destructive technique particularly well-suited to probing the fine details of surfaces or buried interfaces ${ }^{26}$ where the X-rays probe the electron density perpendicular to the surface normal. In this instance, the diffraction is called specular (or longitudinal) elastic scattering, i.e. that the incident and exit wavevectors, $\mathbf{k}_{\mathbf{i}}$ and $\mathbf{k}_{\mathbf{f}}$, respectively, have the same magnitude, and that the angles of incidence and exit, $\theta_{\mathrm{i}}$ and $\theta_{\mathrm{f}}$, respectively, are also equivalent. The X-ray intensity is measured as a function of incidence angle, close to the critical angle, $\theta_{\mathrm{C}}$, for total external reflection, which is typically a combination of Fresnel reflectivity (with a $\left|1 / \mathbf{q}_{\mathbf{z}}\right|^{4}$ dependence) and a fringe pattern (commonly Keissig fringes), due to constructive interference from scattering at the layer interfaces. Here, $\mathbf{q}_{\mathbf{z}}$ is the wavevector momentum transfer along the surface normal. 


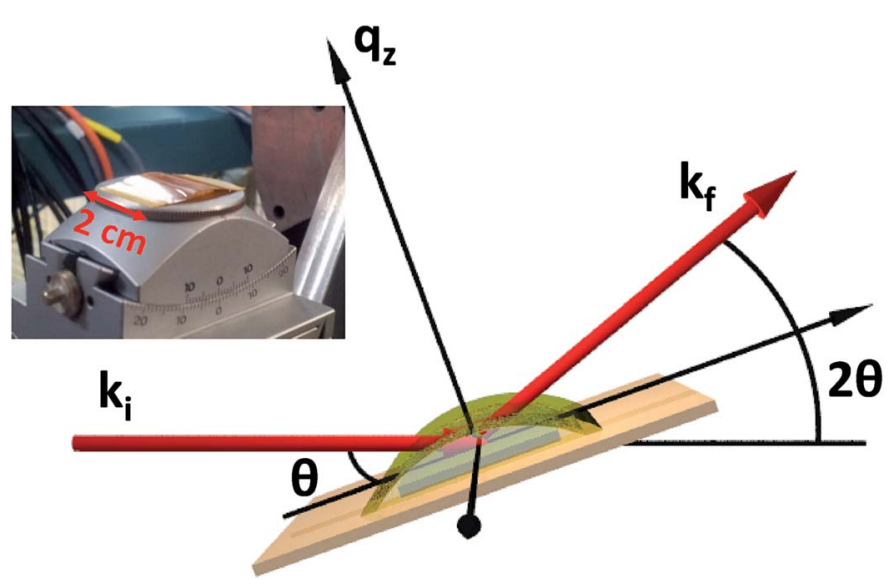

Fig. 1 Schematic of the set-up used in the laboratory characterisation measurements and synchrotron experiments at the ESRF and Diamond Light Sources. Here, the incident and exit wavevectors $\left(\mathbf{k}_{\mathbf{i}}\right.$ and $\left.\mathbf{k}_{\mathbf{f}}\right)$, angle of incidence $(\theta)$, angle with respect to the detector $(2 \theta)$ and the wavevector momentum transfer $\left(q_{z}\right)$ are labelled. The thin layer surface tension cell can be seen over the sample, which is used to hold a fixed volume of water that covers the entire sample during X-ray irradiation. The insert shows a photograph of the cell, the horizontal dimension is noted along the edge of the cell.

$$
\mathbf{q}_{\mathbf{z}}=\mathbf{k}_{\mathbf{f}} \sin \theta_{\mathrm{f}}-\mathbf{k}_{\mathbf{i}} \sin \theta_{\mathrm{i}}=\frac{4 \pi}{\lambda} \sin \theta
$$

In this case, we have used the Parratt recursion method ${ }^{27}$ of calculating transmitted and reflected wave fields, using the GENX computer program, developed by Björck and Andersson, ${ }^{28}$ which fits calculated reflectivity profiles to experimental data, using a differential evolution algorithm that can be optimised to avoid local minima; a common problem encountered when modeling X-ray reflectivity. The variables that are used to construct the electron density profile are the densities of materials $\left(\rho_{\text {substrate }}, \rho_{\mathrm{UO}_{2}}\right)$, the layer thickness $\left(t_{\mathrm{UO}_{2}}\right)$ and the roughness of each interface $\left(\sigma_{\text {substrate }}, \sigma_{\mathrm{UO}_{2}}\right)$, measured as the root mean squared of the fluctuations in the height of the layer. Also included in the model is a top layer of uranium oxide that is lower in electron density than stoichiometric $\mathrm{UO}_{2}$, which we label $\mathrm{UO}_{X}$. This layer is modelled by a series of slices of varying electron

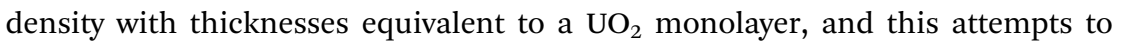
interpret both the topology and hyperstoichiometric progression of uranium oxide. Later, in Table 1 an average $t_{\mathrm{UO}_{x}}$ is given, that represents the mean overall thickness of this top-layer, assuming half the electron density of $\mathrm{UO}_{2}$ (i.e. the average between the bulk value and air).

High resolution X-ray diffraction provides a host of complementary information to describe the structural composition of thin films. In the case of a singlecrystal [001]-UO $\mathrm{UO}_{2}$ film on YSZ, a longitudinal measurement across the (002) Bragg peak gives the average $d$-spacing for the thin film lattice parameter along the surface normal, and the finite thickness broadening of the Bragg peak can be used to calculate the number of scattering planes contributing to the intensity, and therefore the thickness of crystalline $\mathrm{UO}_{2}$. For smooth interfaces, fringes are 
Table 1 Table of parameters used in the fitted calculations to model the experimental reflectivity and high angle diffraction data. All values are in $\AA$, where $t_{\mathrm{UO}_{2}}$ is the thickness of $\mathrm{UO}_{2}$ and $\sigma_{\cup \mathrm{O}_{2}}$ is the root mean squared roughness, both have errors of approximately $1 \AA$, since both high angle XRD and XRR are used to determine these values. $t_{\cup O_{x}}$ and $\sigma_{\cup O_{x}}$ are the thicknesses and roughnesses of the top layer of complex oxide, respectively, and have larger errors of $\sim 2 \AA$

\begin{tabular}{llccc}
\hline Exposure time (s) & $t_{\mathrm{UO}_{2}}$ & $\sigma_{\mathrm{UO}_{2}}$ & $t_{\mathrm{UO}_{X}}$ & $\sigma_{\mathrm{UO}_{X}}$ \\
\hline 0 & 34 & 3 & 10 & 6 \\
30 & 27.5 & 2.5 & 16 & 9 \\
90 & 21 & 7.6 & 20 & 11.5 \\
120 & 13.5 & 11 & 22 & 13
\end{tabular}

also present, similar to those observed in X-ray reflectivity, which give information about the electron density profile. A rocking curve at the Bragg peak position produces an observable intensity from planes that are not perfectly parallel. This gives an indication of the crystal mosaicity and the density of dislocation defects. The rocking curve measurement is made by rotating the sample (varying $\theta$ ), while keeping the detector $(2 \theta)$ fixed, see Fig. 1.

\section{Results}

Using a combination of X-ray reflectivity and high resolution diffraction, it is then possible to build up a full structural picture of the thin film and the substrate/film and film/air interface. Fig. 2 shows the X-ray reflectivity (panel (a)) and high resolution diffraction (panel (b)) from a single-crystal [001]- $\mathrm{UO}_{2}$ film on YSZ, with a nominal thickness of $40 \AA$. The data are shown as open black circles and fitted
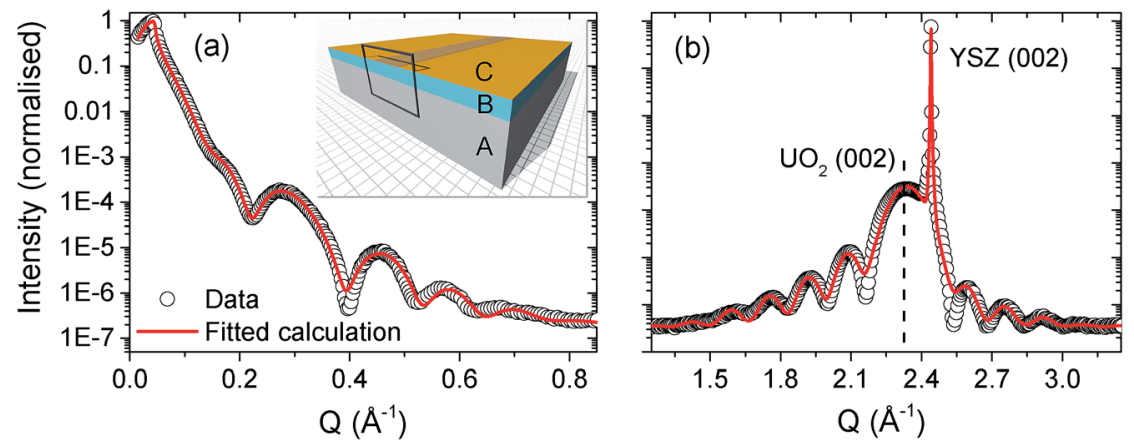

Fig. 2 Panel (a) shows the $X$-ray reflectivity spectrum for a nominal $40 \AA$ [001] $-\cup_{2}$ thin film, grown on YSZ. Panel (b) contains a high resolution diffraction spectrum across the $\mathrm{UO}_{2}(002)$ Bragg peak. The $\mathrm{UO}_{2}(002)$ thin film and YSZ (002) substrate Bragg peaks have been labelled for clarity. The experimental data are the open black circles and the fitted calculations are the solid red lines. The insert in panel (a) shows the model of a pristine $\mathrm{UO}_{2}$ film, labelled B, with a complex surface oxide layer, C, grown on a YSZ substrate, A. The copper coloured strip across the centre of the sample indicates the footprint of the X-ray beam at a low angle and the frame indicates the 2D profile used to indicate the corrosion front in Fig. 4. 
calculations of the reflectivity and the high angle diffraction are represented by solid red lines.

These data were taken at the I07 beamline of the Diamond Light Source. The photon flux at a synchrotron source is between 4 and 5 orders of magnitude greater than a laboratory source and so allows one to probe the reflectivity far further in $\mathbf{q}_{\mathbf{z}}$ and better resolve the fringes at higher angles. It is also ideal to study buried interfaces, which in our case will be the $\mathrm{UO}_{2}$ /water interaction, since we are interested in the potential dissolution of $\mathrm{UO}_{2}$ in groundwater.

Table 1 includes the model parameters used to reproduce the calculations. It is clear that even for a pristine sample, not exposed to heavily oxidising conditions, it is necessary to model the thin film with a $\sim 30 \AA$ crystalline $\mathrm{UO}_{2}$ layer and then a top layer of low electron density oxide. The insert of Fig. 2(a) shows a model of the pristine film, where the substrate layer is labelled $\mathrm{A}$, the $\mathrm{UO}_{2}, \mathrm{~B}$, and the top oxide layer, C. Since we are concerned with the corrosion of $\mathrm{UO}_{2}$ in water, the next step is to repeat these measurements, using synchrotron radiation to simultaneously probe the film structure and to provide the necessary radiolytic products for oxidative dissolution.

In order to probe the change in the uranium oxide film in detail as a function of exposure time, first we set the detector position to the centre of the $\mathrm{UO}_{2}(002)$ Bragg peak and measured the intensity as a function of time. The following data were taken on the 107 beamline at the Diamond Light Source, UK. The beam energy was monochromated to $17.116 \mathrm{keV}$ ( $50 \mathrm{eV}$ below the $\mathrm{U} \mathrm{L}_{3}$ absorption edge), which was then focused in the vertical and horizontal directions to give an approximate beam size at the sample position of $200 \mu \mathrm{m} \times 200 \mu \mathrm{m}$. The incident beam slits were set to $100 \mu \mathrm{m} \times 100 \mu \mathrm{m}$ for all measurements and the scattered photons were detected, using a Pilatus $100 \mathrm{~K}$ detector.

In Fig. 3(a) and (b) we present data at three points in time, $30 \mathrm{~s}, 90 \mathrm{~s}$ and $120 \mathrm{~s}$. The thin layer surface tension cell was filled with Milli-Q ultra pure type 1 water and the incident slits were set to a 200 micron square. The surface was exposed at
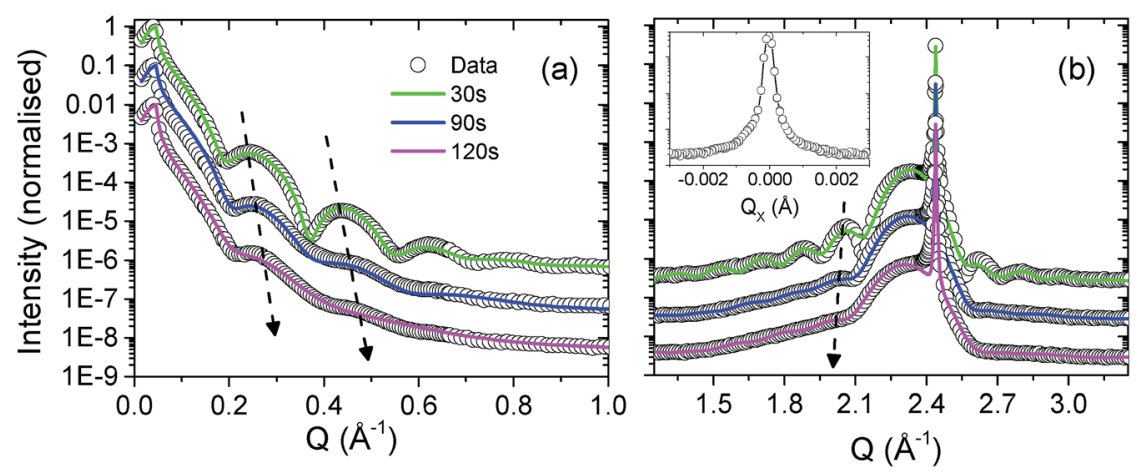

Fig. 3 Panel (a) shows $X$-ray reflectivity data and panel (b) shows high angle diffraction data, measured at exposure times of $30 \mathrm{~s}, 90 \mathrm{~s}$ and $120 \mathrm{~s}$; the experimental data are represented by the open black circles and the fitted calculations by the solid green, blue and magenta lines, respectively. The insert of panel (b) shows the rocking curve of the (002) Bragg peak for the $30 \mathrm{~s}$ exposure. The dashed black arrows indicate an increase in fringe separation as a function of exposure time, which suggests a concomitant loss of material. 
an incident angle of $\sim 0.5^{\circ}$, such that the footprint was approx. $2 \mathrm{~cm}$, more than covering the whole length of the sample. The water was then removed, the slits were closed down to $100 \mu \mathrm{m} \times 100 \mu \mathrm{m}$ and the diffraction spectra were recorded. The water removal was carried out using a pipette, and an $\mathrm{N}_{2}$ gas flow jet was positioned close to the sample surface during measurements. Two simple tests were carried out to confirm the necessary conditions of a surface/water interface and radiation strong enough to drive radiolysis: the first involved exposing the $\mathrm{UO}_{2}$ surface to water for two hours and then measuring the X-ray reflectivity and diffraction profiles; no changes were detected. The second test involved exposing a sample to an intense X-ray beam for an hour with no water present; again, no changes to the scattered intensity were observed.

A calibrated Si photodiode was placed in the beam at the sample position in order to accurately calculate the number of photons per second per unit area incident for a number of slit settings. Together with the beam energy, these values could then be used to calculate the likely number of oxidising species present in the X-ray beam path in the water, specifically, to calculate the number of long lived, oxidising $\mathrm{H}_{2} \mathrm{O}_{2}$ molecules. Fig. 3 shows fitted calculations to the experimental data, based on a structural model of the $\mathrm{UO}_{2}$ film that consists of a layer of crystalline $\mathrm{UO}_{2}$ with the standard bulk density and a surface layer of reduced electron density, labeled $\mathrm{UO}_{X}$. Roughnesses for each of the substrate/ $\mathrm{UO}_{2}\left(\sim 2 \AA\right.$ in each case), $\mathrm{UO}_{2} / \mathrm{UO}_{X}$ and $\mathrm{UO}_{X} /$ water interfaces were also computed. Table 1 summarises all of the parameters and Fig. 4 shows a pictorial representation of the corrosion region at each of the $30 \mathrm{~s}, 90 \mathrm{~s}$ and $120 \mathrm{~s}$ exposures.

Initially, the intensity of the $\mathrm{UO}_{2}(002)$ Bragg peak was measured as a function of the exposure time. This is not precisely a measure of the dissolution, since a decrease in intensity will also result from a surface roughening and oxidation, but it does provide a good estimate for the rate of change of the interface structure. This measurement was carried out for thin film samples of [001] (polar surface), [110] and [111] orientations (non-polar surfaces), which due to their different surface energies and water adsorption energies are expected to have significant impact on the rate of dissolution. However, contrary to this assumption, within the errors of this experiment, we saw no evidence to suggest that this may play a significant role. This confirmed earlier measurements carried out at an incident energy of $15 \mathrm{keV}$ at the XMaS beamline, where also the experiment was repeated under alkaline $(\mathrm{pH} \sim 11)$ and acidic $(\mathrm{pH} \sim 2)$ conditions. As expected, in the case of increased acidity, the rate of corrosion dramatically increased, whereas under

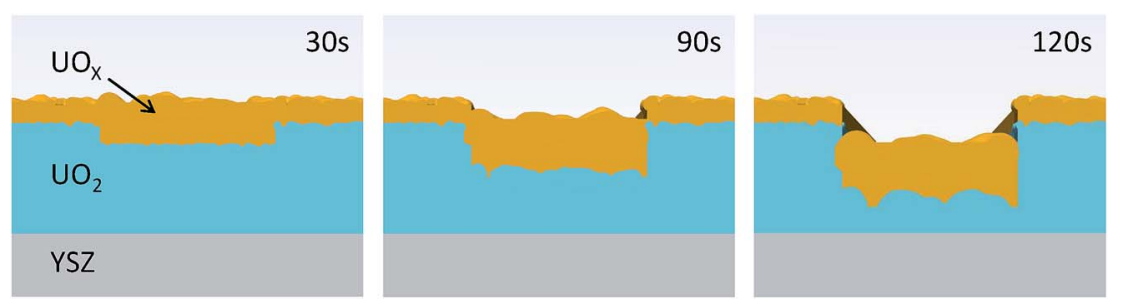

Fig. 4 Pictorial representation of the increase in roughness and $\cup O_{X}$ thickness, and the amount of dissolution as the surface undergoes corrosion. 
heavily alkaline conditions the rate of change in the $\mathrm{UO}_{2}$ Bragg peak intensity all but stopped, i.e. dissolution was halted.

Furthermore, since we were also interested in any potential surface enhancement of the corrosion by the photocatalytic process, we measured the rate of change in the Bragg peak intensity before (17.116 keV), at (17.166 keV) and after (17.216 keV) the uranium $\mathrm{L}_{3}$ absorption edge. There is a huge resonant enhancement in the number of electrons excited to the continuum at such an absorption edge, particularly for the $\mathrm{U}_{3}$, so this ought to have a pronounced effect on any possible photocatalytic process, however, we did not observe any difference in the rate of the decrease in the Bragg peak intensity, within experimental errors.

\section{Discussion}

So far we have seen experimentally the effect of radiolytically driven oxidation and dissolution of the $\mathrm{UO}_{2}$ surface, but it is also possible to calculate the quantity of radiolysis products from first principles, based on the energy and flux of the X-ray source incident on a known water thickness. In the first instance, we are making the same assumption as the vast majority of the literature, that the longest lived and dominant oxidising product is hydrogen peroxide. ${ }^{6,23}$ Here, we have $\sim 1 \times$ $10^{12}$ photons per s of $17.116 \mathrm{keV} X$-rays incident on a $0.5 \mathrm{~mm}$ thick water layer on the $\mathrm{UO}_{2}$ film surface. The $G$-value, the number of molecules of reactant consumed or product formed (in our case) per unit of incident energy absorbed, is 0.6 molecules of $\mathrm{H}_{2} \mathrm{O}_{2} / 100 \mathrm{eV}$.

For this thickness of water at this photon energy, the transmission of photons to the surface is $\sim 59 \%$, which results in $\sim 7 \times 10^{-11}$ moles $\mathrm{H}_{2} \mathrm{O}_{2}$ produced. Since the volume of the water exposed to the beam is approx. $200 \mu \mathrm{m}$ wide, $0.5 \mathrm{~mm}$ high and $1 \mathrm{~cm}$ long, i.e. $0.001 \mathrm{~cm}^{3}$, the $\mathrm{H}_{2} \mathrm{O}_{2}$ concentration increases by $\sim 7 \times 10^{-5} \mathrm{M}$ per second. The equilibrium concentration is in the region of $1 \times 10^{-4} \mathrm{M}$, which means that it is reached almost instantly, relative to the timescales of this experiment. The question then arises - is $\mathrm{H}_{2} \mathrm{O}_{2}$ alone enough to drive the changes that we are observing in our experiment? This is the general assumption purported by the literature. ${ }^{6,23}$

Fig. 5 may provide a clue. Panel (a) is a scanning electron microscopy image, obtained using a Zeiss Sigma FEG-SEM; utilising secondary electron detection with electron gun settings of $10 \mathrm{kV}$ and $30 \mu \mathrm{A}$. The image shows the area of the sample that has been exposed to the beam. This area is heavily corroded and so is not as conductive as the surrounding $\mathrm{UO}_{2}$ film. What is observed here is the resultant charging of the corroded region. There is one particularly remarkable feature and that is that the width of the corrosion track is $100 \mu \mathrm{m}$, which is precisely the slit settings used in the experiment. During the duration of the experiment, one might imagine that due to diffusion of the $\mathrm{H}_{2} \mathrm{O}_{2}$ species there would be a far wider area of corroded material. Panels (b) and (c) of Fig. 5 represent a finite element model, including (i) a short-lived species, which is confined to the radiolysis volume within the beam path, or one produced only at the sample surface and (ii) a long-lived species (such as $\mathrm{H}_{2} \mathrm{O}_{2}$ ) subject to diffusive transport through the water layer in order to determine the likely footprint of corrosion. 

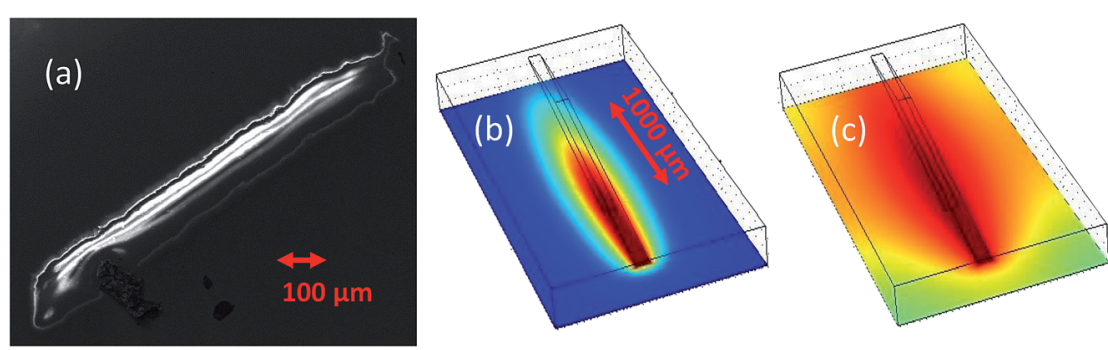

Fig. 5 Panel (a) shows an SEM image of a corroded $\mathrm{UO}_{2}$ single-crystal thin film, measured at the $\mathrm{UO}_{2}$ Bragg peak position for $500 \mathrm{~s}$ (an incident angle of about $7^{\circ}$ ) with $100 \mu \mathrm{m}$ vertical and horizontal slit settings. Panels (b) and (c) are images representing radiolysis product concentrations close to the beam footprint after $500 \mathrm{~s}$, as calculated using finite element modeling, based on diffusion from the beam volume into the bulk liquid for shortand long-lived species, respectively, with the latter being the representative of $\mathrm{H}_{2} \mathrm{O}_{2}$.

It is clear that a long-lived species, subject to a bulk diffusion, cannot be solely responsible, so this suggests that the corrosion, which is restricted to the beam footprint, is driven by interactions at the surface. There are several candidate propositions that can be explored, for example, it might be due to a photocatalytic

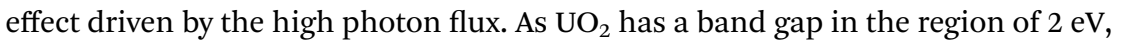
the high flux of X-rays may result in the production of electron-hole pairs, that can further enhance the formation of reactive oxygen species. To test this theory we measured the rate of corrosion at a range of X-ray energies spanning the uranium $\mathrm{L}_{3}$ absorption edge. Crossing through this edge enhances the number of electrons in the valence band, and thus would increase the oxidant species produced via photocatalysis. However, on passing through the $\mathrm{U}_{3}$ edge, no statistically significant increase in the corrosion rate was observed, indicating that in this case a photocatalytic process is not responsible. While it is unclear why we observe this surface enhanced corrosion, other possibilities may include a significant concentration of short-lived oxidising species, such as $\mathrm{OH}$ radicals. Due to the extremely short-lived nature of such species, diffusion outside of the beam footprint would prove unlikely.

\section{Conclusions}

In these experiments we have demonstrated that we can induce significant oxidation and further, dissolution of a $\mathrm{UO}_{2}$ surface, by using an intense beam of $\mathrm{X}$-rays, mimicking the radiation fields found at the surface of spent nuclear fuel. Both the X-rays and the water interface are essential ingredients for these changes. We have been able to measure variations in the electron density, surface roughness and rate of dissolution of a radiolysis driven corrosion front in a nuclear fuel material at the Ångström length-scale.

There still remain some open questions regarding the precise mechanism for the observed corrosion, which seems unlikely to be due to hydrogen peroxide alone and may include a more complex surface effect. This could have significant consequences for previous research that has predominantly relied on this assumption to simulate the conditions driven by radiation fields in real SNF. ${ }^{6,23}$ 
This technique, using thin $(<100 \AA)$ epitaxial films of $\mathrm{UO}_{2}$ gives unprecedented (sub-nanometer) surface sensitivity, and in the future, paves the way for a new set of experiments, using synchrotron X-rays in a series of source-probe measurements, as complexities in fuel structure, cladding and groundwater composition can be incorporated.

\section{Acknowledgements}

The authors wish to thank Paul Thompson and Laura Glaubes for their assistance in the first experiments on this project at the XMaS beamline BM28, ESRF. Ross Springell would like to thank the Royal Commission for the Exhibition of 1851 for the receipt of a research fellowship. The authors would also like to thank the EPSRC for funding the DISTINCTIVE consortium grant on nuclear waste.

\section{References}

1 A. Corner, D. Venables, A. Spence, W. Poortinga, C. Demski and N. F. Pidgeon, Energy Policy, 2011, 39, 4823-4833.

2 C. Pescatore and A. Vari, J. Risk Res., 2006, 9, 13-40.

3 U. Strandberg and M. Andren, J. Risk Res., 2009, 12, 879-895.

4 D. W. Shoesmith, Nuclear Waste Management Organisation, 2007, Report no. NWMO TR-2007-03.

5 T. E. Eriksen, D. W. Shoesmith and M. Jonsson, J. Nucl. Mater., 2012, 420, 409423.

6 D. W. Shoesmith, J. Nucl. Mater., 2000, 282, 1-31.

7 H. He, M. Broczkowski, K. O'Neil, D. Ofori, O. Semenikhin and D. Shoesmith, Nuclear Waste Management Organisation, 2012, NWMO TR-2012-09.

8 F. Garisto, D. Barber, E. Chen, A. Inglot and C. Morrison, Nuclear Waste Management Organisation, 2009, NWMO TR-2009-27.

9 S. Sunder, G. D. Boyer and N. H. Miller, J. Nucl. Mater., 2003, 322, 163-169.

10 D. W. Shoesmith and S. Sunder, SKB Technical Report, 1991, SKB-TR-91-63.

11 M. I. Ojovan and W. E. Lee, An Introduction to Nuclear Waste Immobilisation, Elsevier, 2005, vol. 1.

12 E. Ekeroth, O. Roth and M. Jonsson, J. Nucl. Mater., 2006, 355, 38-46.

13 C. Corbel, G. Sattonnay, S. Guilbert, F. Garrido, M.-F. Barthe and C. Jegou, J. Nucl. Mater., 2006, 348, 117.

14 J. Spinks and R. Woods, An Introduction to Radiation Chemistry, John Wiley and Sons, Inc., New York, 3rd edn, 1990.

15 S. LeCaër, Water, 2011, 3, 235-253.

16 N. Rauff-Nisthar, C. Boxall, I. Farnan, Z. Hiezl, W. Lee, C. Perkins and R. Wilbraham, Corrosion in Nuclear Energy Systems: From Cradle to Grave, ECS Trans., 2013, 53, 95-104.

17 V. Čuba, V. Múčka and M. Pospíšil, in Radiation Induced Corrosion of Nuclear Fuel, ed. D. S. T. Revankar, 2012, pp. 27-52.

18 F. Miserque, T. Gouder, D. Wegen and P. Bottomley, J. Nucl. Mater., 2001, 298, 280-290.

19 A. Seibert, D. Wegen, T. Gouder, J. Ramer, T. Wiss and J. P. Glatz, J. Nucl. Mater., 2011, 419, 112-121. 
20 B. Muzeau, C. Jagou, F. Delaunay, V. Broudic, A. Brevet, H. Catalette, E. Simoni and C. Corbel, J. Alloys Compd., 2009, 467, 578-589.

21 M. G. Bailey, L. H. Johnson and D. W. Shoesmith, Corros. Sci., 1985, 25, 233238.

22 S. Sunder, G. D. Boyer and N. H. Miller, J. Nucl. Mater., 1997, 244, 66-74.

23 S. Sunder, N. H. Miller and D. W. Shoesmith, Corros. Sci., 2004, 46, 1095-1111.

24 M. M. Strehle, B. J. Heuser, M. S. Elbakhshwan, X. Han, D. J. Gennardo, H. K. Pappas and H. Ju, Thin Solid Films, 2012, 520, 5616-5626.

25 S. D. Brown, L. Bouchenoire, D. Bowyer, J. Kervin, D. Laundy, M. J. Longfield, D. Mannix, D. F. Paul, A. Stunault, P. Thompson, M. J. Cooper, C. A. Lucas and W. G. Stirling, J. Synchrotron Radiat., 2001, 8, 1172-1181.

26 P. F. Fewster, Rep. Prog. Phys., 1996, 59, 1339.

27 L. G. Parratt, Phys. Rev., 1954, 95, 359.

28 M. Björck and G. Andersson, J. Appl. Crystallogr., 2007, 40, 1174. 